\title{
SEGURIDAD ALIMENTARIA EN HOGARES \\ DE ACANDÍ, DARIÉN CARIBE COLOMBIANO: \\ EL APORTE DEL CARACOL CITTARIUM PICA "LA CIGUA"
}

\author{
HOUSEHOLD FOOD SECURITY IN ACANDÍ, \\ DARIEN CARIBBEAN COLOMBIAN: THE CONTRIBUTION \\ OF SNAIL CITTARIUM PICA "THE WEST INDIAN TOP SHELL"
}

\author{
Gustavo Lozano C. (1), Alejandro Estrada R. $(1,2)$ \\ (1) Escuela de Nutrición y Dietética, Universidad de Antioquia, Colombia \\ (2) Grupo de Demografía y Salud, Facultad Nacional de Salud Pública, Universidad de Antioquia, Colombia
}

\begin{abstract}
Household food security in Capurgana and Sapzurro and the association with the collection and the consumption of the snail Cittarium pica is described. A cross sectional-study was carried out in a representative sample of 126 households obtained using a probabilistic sampling. A food security perception scale was applied to determine its severity, and other characteristics were collected through a survey. Statistical analysis was done using the chi-square test, the $U$ mann whitney test, odds ratio along with its confidence intervals and the correspondence multiple analysis. More than half $(54,8 \%)$ of households were in food insecurity. Food insecurity was significantly associated with low income ( $p=0,0003)$, direct collection of the snail $(p=0,0178)$, education level of the head of the household $(p=0,0014)$ and the money spent on food $(p=0,0000)$. The current use of the snail does not contribute to the household food security
\end{abstract}

Key words: Food security, Cittarium pica, Capurganá, Sapzurro, Colombia.

Este trabajo fue recibido el 20 de Abril de 2008 y aceptado para ser publicado el 20 de Septiembre de 2008

\section{INTRODUCCIÓN}

Debido a la sobreexplotación de los recursos naturales, la zona del Darién Caribe colombiano afronta actualmente un deterioro medio ambiental que ha ocasionado la destrucción del hábitat y la extinción de especies, generando así la pérdida de recursos que contribuían a la seguridad alimentaria y nutricional de sus pobladores.

Por seguridad alimentaria se entenderá lo expuesto en la Cumbre Mundial sobre la Alimentación, convocada por la FAO en 1996, donde se estableció que "Existe seguridad alimentaria cuando todas las personas en todo momento tienen acceso económico y físico a suficientes alimentos inocuos y nutritivos para satisfacer sus necesidades alimentarias y preferencias en cuanto alimentos a fin de llevar una vida sana y activa" (1). Concepto sobre el cual se basa la Política Nacional de Seguridad Alimentaria de Colombia, que tiene como principios el derecho a la alimentación, equidad, sostenibilidad, responsabilidad y el respeto a la diversidad cultural (2).

En el municipio de Acandí, Darién Caribe colombiano, la seguridad alimentaria se encuentra comprometida debido a aspectos como el descenso de la producción agrícola, la poca participación de la población en actividades productivas, la falta de infraestructura para cadenas de frío y otras formas de conservación de alimentos, la falta de ingresos monetarios y la dependencia de compra en mercados externos al municipio (3).

La población de Acandí, presenta problemas de malnutrición, así lo evidencia la tasa de muerte por desnutrición en los niños menores de cinco años en el periodo 1998 - 2000, la cual fue de 4188,5 por cada 100000 nacidos vivos (4). Así mismo, el departamento de Chocó, al cual pertenece el municipio de Acandí, mostró una prevalencia de retraso en el crecimiento del $11.7 \%$ y una prevalencia de sobrepeso de $10.8 \%$, en los 
niños de 10 a 17 años. Para las personas de 18 a 64 años se encontró una prevalencia de sobrepeso de $31.1 \%$ y de obesidad de $16.2 \%$ (5).

En las localidades de Capurganá y Sapzurro del municipio de Acandí, las cuales conforman el área geográfica del presente estudio, el caracol de mar Cittarium pica, conocido con el nombre de "cigua", es un alimento popular y tradicional.

El comportamiento sedentario y lento crecimiento de $\mathrm{C}$. pica contribuyen a que sus poblaciones sean vulnerables a la sobrepesca. Actualmente, C. pica está clasificada en la categoría vulnerable (VU) según la resolución 0584 del 26 de Junio de 2002 del Ministerio del Medio Ambiente de Colombia (6), y su vulnerabilidad en las localidades del estudio fue confirmada recientemente (7). A pesar de su sobreexplotación, C. pica sigue siendo un recurso socio-económicamente importante en la zona (8) y por lo tanto juega un papel en la seguridad alimentaria de sus pobladores.

En las localidades de Capurganá y Sapzurro, del municipio de Acandí, se han realizado algunos estudios sobre aspectos bioecológicos y socioculturales de C. pica (7-9), pero aún se requieren datos sobre su valor nutricional, su consumo, su contribución a la economía del hogar y su relación con la seguridad alimentaria de los hogares.

Este trabajo permitió describir la seguridad alimentaria en hogares de las localidades de Capurganá y Sapzurro y su relación con la utilización del recurso C. pica.

\section{SUJETOS Y MÉTODO}

Tipo de estudio: descriptivo transversal.

Población y muestra: la población estuvo constituida por los hogares de los corregimientos de Capurganá y Sapzurro del municipio de Acandí, el cual se encuentra ubicado en el extremo norte del departamento del Chocó, Darién Caribe colombiano en limites con Panamá.

Se obtuvo una muestra representativa y probabilística, calculada con una confianza del 95\%, un error de muestreo del $5 \%$, y una probabilidad de encontrar familias que consumen C. pica del $35 \%$, la que quedó constituida por 126 hogares. El muestreo fue aleatorio estratificado por localidad.

Se aplicó una prueba piloto a una encuesta que contenía variables sobre información demográfica (escolaridad, edad, sexo), económica (ocupación, ingresos, gastos en alimentación), autoconsumo, seguridad alimentaria y el recurso C. pica (captura, comercialización y consumo). Esta prueba, permitió verificar la consistencia de las preguntas, los tiempos de respuesta y la comprensión de las preguntas por parte de los encuestados. Una vez se ajustó la encuesta de acuerdo a las observaciones encontradas, se procedió a su aplicación por parte de dos integrantes del grupo de investigación.

Para evaluar la seguridad alimentaria en hogares, se utilizó la escala que Álvarez y cols emplearon en la Encuesta Nacional de la Situación Nutricional en Colombia 2005 (5); escala validada por la autora en hogares urbanos y rurales del departamento de Antioquia, Colombia (10). Esta escala había sido validada y adaptada previamente en hogares pobres de Caracas por Lorenzana (11), quien se basó en la escala original utilizada por Wehler, Scott y Anderson (12).

La escala utilizada estaba constituida por 12 ítems relacionados con la disponibilidad de dinero para la compra de alimentos, la disminución de comidas en el hogar por falta de dinero y la experiencia de hambre de alguno de los integrantes del hogar. La frecuencia de ocurrencia de cada ítem se codificó de la siguiente manera: "siempre", tres puntos; "algunas veces", dos puntos; "rara vez", un punto y "nunca", cero puntos. Esta codificación permitió generar para hogares con integrantes menores de 18 años, un puntaje de inseguridad alimentaria con un rango entre 0 y 36 puntos, a partir del cual los hogares se clasificaron en cuatro categorías: 0 puntos como "seguros"; 1-12, "inseguridad alimentaria leve"; 13-24, "inseguridad alimentaria moderada"; y 25 ó más, "inseguridad alimentaria severa". Para los hogares sin integrantes menores de 18 años, el puntaje máximo obtenido es de 21 , estos se clasificaron así: 0 puntos como "seguros"; 1-7, "inseguridad alimentaria leve"; 8-14, "inseguridad alimentaria moderada", y 15 o más, "inseguridad alimentaria severa".

Criterios de inclusión: se encuestó el jefe del hogar o quien hiciera las veces en el momento de la encuesta, en todos los casos fue una persona mayor de 18 años.

Aspectos éticos: las personas participaron de manera voluntaria en las diferentes actividades y se les comunicó que la información suministrada por ellos sería confidencial y que sólo se utilizaría para efectos de la investigación (consentimiento verbal). Dado el tipo de estudio y las técnicas utilizadas en el mismo, esta investigación cumplió con los principios básicos de la declaración de Helsinki (13) y se concibió sin riesgo para los participantes según la resolución 008430 de 1993 del Ministerio de Salud de Colombia (14). La investigación fue aprobada por el Comité Central de Bioética de la Universidad de Antioquia.

Análisis de los datos: el análisis estadístico se realizó a partir de medidas descriptivas como la media, desviación estándar, mediana y porcentajes, con sus respectivos intervalos de confianza. Se aplicaron las pruebas de Kolmogorov-smirnov y el test de Levene para 
determinar la normalidad de las variables y la homogeneidad de varianzas respectivamente. Se utilizó la prueba de chi cuadrado y la prueba U de Mann Whitney para relacionar características de localidad, demográficas, de seguridad alimentaria y del recurso C. pica, además del odds ratio con sus respectivos intervalos de confianza. Asimismo, se efectuó un análisis de correspondencias múltiples para establecer perfiles entre las características, este último es una técnica descriptiva exploratoria que cuantifica datos categóricos, resumiendo la información de las variables introducidas en una serie de factores (ejes) los cuales explican la variabilidad observada en los datos, mostrándolos en un gráfico en el cual se exponen patrones que sugieren hipótesis $(15,16)$. Valores de $p<0,05$ se entendieron como significativos. Para el análisis de los datos se utilizó el software Spss versión 14 (Spss Inc. Chicago).

\section{RESULTADOS}

El 51,4\% de los habitantes de Capurganá y Sapzurro fueron de sexo masculino, el grupo de edad con el porcentaje más alto fue el de 0-4 años, seguido del de 10-14 años. El promedio de edad fue de 25,3 $\pm 18,7$ (ic $_{95 \%} 23,5 ; 27,1$ ) para Capurganá y de $29,4 \pm 19,8$ para Sapzurro ( $\left.\mathrm{ic}_{95 \%} 25,4 ; 33,4\right)$. Para las dos localidades el

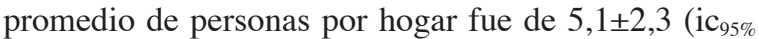
$4,9 ; 5,3)$, con un mayor valor en Capurganá 5,2 2 ,2 (ic is\% $\left._{9,0}, 5,5\right)$ (tabla 1).

Las ocupaciones manifestadas principalmente por los habitantes de las dos localidades fueron: estudiante $(29,4 \%)$, ama de casa $(24,8 \%)$ y oficios varios en su mayoría relacionados con el turismo (19,8\%), la agricultura representó el $4.4 \%$ de las ocupaciones.

La mayoría de los hogares de las dos localidades habitaban en viviendas propias $(65,9 \%)$, con una mayor proporción de estas en Sapzurro (89,3\%) que en Capurganá ( $\mathrm{p}=0,0030)$.

La cantidad de dinero que ingresó mensualmente al 79,2\% de los hogares fue menor o igual a un salario mínimo (valor del 2006, \$408 000) (tabla 1). Un 25,8\% de los hogares manifestó ingresos provenientes de la pesca.

En lo concerniente a la cantidad de dinero que se destinaba para alimentación, se halló que este gasto representó más del $80 \%$ de los ingresos totales mensuales de los hogares. En Capurganá se destinó en promedio

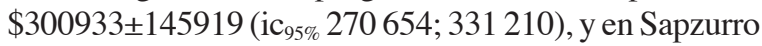
$\$ 338520 \pm 152820$ (ic $95 \% 279565$; 397474) (tabla 1).

Para la seguridad alimentaria se halló que un poco más de la mitad de los hogares se percibieron en inseguridad $\left(54,8 \%\right.$, ic c $_{95 \%} 46,5 \%$; 63,5\%). Por localidad, el $56,1 \%\left(\right.$ ic $\left._{95 \%} 46,3 ; 66,0\right)$ de los hogares de Capurganá y el
$50 \%\left(\mathrm{ic}_{95 \%} 31,4 ; 63,5\right)$ de los de Sapzurro se percibieron en esta clasificación (tabla 1).

En lo que respecta al recurso $\mathrm{C}$ pica, el $69 \%$ (ic $_{95 \%}$ $60,1 \% ; 76,8 \%$ ) de los hogares nunca lo capturaban, este comportamiento fue de un $70,4 \%$ (ic is\% $_{95} 61,3 ; 79,5$ ) y $64,3 \%$ (ic 95\% $\left._{4} 46,5 ; 82,1\right)$ para Capurganá y Sapzurro respectivamente. Dentro de los hogares que capturaban, estos lo hacían esencialmente en los meses de septiembre y octubre.

El 53,2\% (ic 95\% $_{44,4 \%}$; 61,9\%) de los hogares manifestaron consumir C. pica, resultando ser más alta la proporción de consumo en Sapzurro $(\mathrm{p}=0.0282)$ (tabla 2). La frecuencia semanal de consumo fue baja (menor o igual a una vez por semana) para ambas localidades.

En cuanto a la cantidad de C. pica consumida, el $71 \%$ de los hogares manifestó que era menor o igual a 5 libras anuales y el $14,5 \%$ de 6 a 10 libras anuales (tabla 2).

La forma como C. pica se obtenía fue principalmente por recolecta directa en el 58,5\% de los hogares y por compra en el $36,9 \%$. Los datos por localidad no mostraron diferencias significativas para recolecta directa $(\mathrm{p}=0,3916)$, ni para compra $(\mathrm{p}=0,8389)$ (tabla 2$)$.

Según la seguridad alimentaria de los hogares de estas localidades (tabla 3), se encontró que dentro de los hogares inseguros, el 13,4\% se encontraban en hacinamiento, porcentaje que fue un poco más elevado que en los hogares seguros $(\mathrm{p}=0,8783)$.

El 91,3\% de los hogares inseguros, presentaron ingresos menores o iguales a un salario mínimo ( $\$ 408000$ valor año 2006), porcentaje más alto que en los hogares seguros $(\mathrm{p}=0,0003)$. Se evidenció que tener bajos ingresos aumenta 5,6 veces la posibilidad de inseguridad alimentaria (tabla 4). Al comparar ambos tipos de hogares no se encontraron diferencias en cuanto a la alimentación subsidiada ( $\mathrm{p}=0,8295)$, ni al consumo de C. pica en el hogar $(\mathrm{p}=0,3345)$.

Los hogares inseguros, destinaban en promedio menos dinero para la compra de alimentos $(p=0,0000)$ que los hogares seguros. En los hogares inseguros el aporte de ingresos fue realizado en mayor proporción por un solo miembro del hogar $(\mathrm{p}=0,0138)$, y sus jefes de hogar tenían menos años de escolaridad $(\mathrm{p}=0,0014)$ en comparación con los hogares seguros (tabla 3).

En cuanto a esta última característica, se evidenció que con un mayor número de años de escolaridad del jefe del hogar se disminuye la posibilidad de tener un hogar en inseguridad alimentaria $\left(\mathrm{OR}=0,86, \mathrm{IC}_{95 \%} 0,76 ; 0,97\right)$ (tabla 4). En los hogares que manifestaron consumir C. pica se encontró diferencias en cuanto a la forma de adquisición, mostrando que la recolecta directa fue más alta en los hogares inseguros $(72,7 \%)$ que en los 


\section{TABLA 1}

\section{Características sociodemográficas y de seguridad alimentaria de los hogares de las localidades de Capurganá y Sapzurro}

\begin{tabular}{|c|c|c|c|c|}
\hline Características & Capurganá & Sapzurro & Total & $\mathbf{p}$ \\
\hline \multirow[t]{2}{*}{ Número de personas* } & $n=414$ & $n=97$ & $\mathrm{n}=511$ & \\
\hline & $5,2 \pm 2,2(5)$ & $4,42 \pm 2,3(3)$ & $5,1 \pm 2,3(5)$ & $0,000 * *$ \\
\hline \multirow[t]{2}{*}{ Hasta que año estudio* } & $n=414$ & $\mathrm{n}=97$ & $\mathrm{n}=511$ & \\
\hline & $5,2 \pm 3,4(5)$ & $5,7 \pm 3,6(5)$ & $5,3 \pm 3,4(5)$ & $0,4703 * *$ \\
\hline Tipo de familia (\%) & $\mathrm{n}=98$ & $n=28$ & $n=126$ & \\
\hline Unipersonal & 6,1 & 3,6 & 5,6 & \\
\hline Nuclear uniparental padre & 3,1 & 0 & 2,4 & \\
\hline Nuclear uniparental madre & 7,1 & 14,3 & 8,7 & \\
\hline Nuclear pareja sin hijos & 5,1 & 25 & 9,5 & $0,0208 * * *$ \\
\hline Nuclear completa & 34,7 & 21,4 & 31,7 & \\
\hline Extensa & 8,2 & 0 & 6,3 & \\
\hline Mixta & 35,7 & 35,7 & 35,7 & \\
\hline Hacinamiento (\%) & $\mathrm{n}=95$ & $\mathrm{n}=28$ & $\mathrm{n}=123$ & \\
\hline $\mathrm{Si}$ & 15,8 & 3,6 & 13,0 & \\
\hline No & 84,2 & 96,4 & 87,0 & $0,1162 * * *$ \\
\hline \multicolumn{5}{|l|}{ Número de personas que } \\
\hline aportan ingresos (\%) & $\mathrm{n}=96$ & $\mathrm{n}=28$ & $\mathrm{n}=124$ & \\
\hline 1 & 49 & 67,9 & 53,2 & \\
\hline 2 & 35,4 & 32,1 & 34,7 & $0,1167 * * *$ \\
\hline 3 & 9,4 & 0 & 7,3 & \\
\hline 4 & 6,3 & 0 & 4,8 & \\
\hline Ingresos mensuales & $\mathrm{n}=97$ & $\mathrm{n}=28$ & $\mathrm{n}=125$ & \\
\hline Entre 0 y $\$ 200.000$ & 32,0 & 35,7 & 32,8 & \\
\hline Entre $\$ 201.000$ y $\$ 408.000$ & 47,4 & 42,9 & 46,4 & \\
\hline Entre $\$ 409.000$ y $\$ 600.000$ & 13,4 & 10,7 & 12,8 & $0.8746 * * *$ \\
\hline Entre $\$ 601.000$ y $\$ 800.000$ & 3,1 & 7,1 & 4,0 & \\
\hline Más de $\$ 800.000$ & 4,1 & 3,6 & 4,0 & \\
\hline \multicolumn{5}{|l|}{ Cantidad de dinero } \\
\hline \multirow[t]{3}{*}{ destinado a alimentación* } & $\mathrm{n}=98$ & $\mathrm{n}=28$ & $\mathrm{n}=126$ & \\
\hline & $300933 \pm 145919$ & $338520 \pm 152820$ & $309175 \pm 147600$ & $0,2773 * *$ \\
\hline & $(300000)$ & $(300000)$ & $(300000)$ & \\
\hline Producción de alimentos & $\mathrm{n}=97$ & $\mathrm{n}=28$ & $\mathrm{n}=125$ & \\
\hline $\mathrm{Si}$ & 23,7 & 35,7 & 26,4 & \\
\hline No & 76,3 & 64,3 & 73,6 & $0,2043 * * *$ \\
\hline Recibe alimentación subsidiada & $\mathrm{n}=98$ & $\mathrm{n}=28$ & $\mathrm{n}=126$ & \\
\hline $\mathrm{Si}$ & 26,5 & 21,4 & 25,4 & \\
\hline No & 73,5 & 78,6 & 74,6 & $0,5844 * * *$ \\
\hline Seguridad alimentaria & $\mathrm{n}=98$ & $\mathrm{n}=28$ & $\mathrm{n}=126$ & \\
\hline Seguro & 43,9 & 50,0 & 45,2 & \\
\hline Inseguro & 56,1 & 50,0 & 54,8 & $0,5659 * * *$ \\
\hline
\end{tabular}


TABLA 2

Características de la captura y el consumo de $\mathbf{C}$. pica en las localidades de Capurganá y Sapzurro

\begin{tabular}{|c|c|c|c|c|c|c|}
\hline \multirow[t]{2}{*}{ Características } & \multicolumn{2}{|c|}{ Capurganá } & \multicolumn{2}{|c|}{ Sapzurro } & \multicolumn{2}{|c|}{ Total } \\
\hline & $\mathbf{n}$ & $\%$ & $\mathbf{n}$ & $\%$ & $\mathbf{n}$ & $\%$ \\
\hline Frecuencia de captura de C. pica & \multicolumn{2}{|c|}{$\mathrm{n}=98$} & \multicolumn{2}{|c|}{$\mathrm{n}=28$} & \multicolumn{2}{|c|}{$\mathrm{n}=126$} \\
\hline Nunca & 69 & 70,4 & 18 & 64,3 & 87 & 69,0 \\
\hline \multicolumn{7}{|l|}{ Ocasionalmente en época de } \\
\hline C. pica (Septiembre-Octubre) & 21 & 21,4 & 5 & 17,9 & 26 & 20,6 \\
\hline \multicolumn{7}{|l|}{ Frecuentemente en época de } \\
\hline C. pica (Septiembre-Octubre) & 8 & 8,1 & 4 & 14,3 & 12 & 9,5 \\
\hline Ocasionalmente en otra época & 0 & 0,0 & 1 & 3,6 & 1 & 1,6 \\
\hline Libras capturadas al año & \multicolumn{2}{|c|}{$\mathrm{n}=30$} & \multicolumn{2}{|c|}{$\mathrm{n}=9$} & \multicolumn{2}{|c|}{$\mathrm{n}=39$} \\
\hline$<=$ de 5 lbs al año & 13 & 43,3 & 5 & 55,6 & 18 & 46,2 \\
\hline $6-10 \mathrm{lbs}$ & 8 & 26,7 & 0 & 0,0 & 8 & 20,5 \\
\hline $11-20 \mathrm{lbs}$ & 3 & 10,0 & 1 & 11,1 & 4 & 10,3 \\
\hline $31-50 \mathrm{lbs}$ & 3 & 10,0 & 2 & 22,2 & 5 & 12,8 \\
\hline$>50 \mathrm{lbs}$ & 3 & 10,0 & 1 & 11,1 & 4 & 10,3 \\
\hline Se consume C. pica en el hogar & \multicolumn{2}{|c|}{$\mathrm{n}=98$} & \multicolumn{2}{|c|}{$\mathrm{n}=28$} & \multicolumn{2}{|c|}{$n=126$} \\
\hline $\mathrm{Si}$ & 47 & 48,0 & 20 & 71,4 & 67 & 53,2 \\
\hline No & 51 & 52,0 & 8 & 28,6 & 59 & 46,8 \\
\hline Cantidad de C. pica consumida al año & \multicolumn{2}{|c|}{$\mathrm{n}=49$} & \multicolumn{2}{|c|}{$\mathrm{n}=20$} & \multicolumn{2}{|c|}{$\mathrm{n}=69$} \\
\hline$<=$ de 5 lbs al año & 33 & 67,3 & 16 & 80,0 & 49 & 71,0 \\
\hline $6-10 \mathrm{lbs}$ & 10 & 20,4 & 0 & 0,0 & 10 & 14,5 \\
\hline $11-20 \mathrm{lbs}$ & 4 & 8,2 & 1 & 5,0 & 5 & 7,2 \\
\hline $21-30 \mathrm{lbs}$ & 0 & 0,0 & 2 & 10,0 & 2 & 2,9 \\
\hline $1-50 \mathrm{lbs}$ & 1 & 2,0 & 1 & 5,0 & 2 & 2,9 \\
\hline$>50 \mathrm{lbs}$ & 1 & 2,0 & 0 & 0,0 & 1 & 1,4 \\
\hline \multicolumn{7}{|l|}{ Forma como se adquiere C. pica en } \\
\hline los hogares que la consumen & \multicolumn{2}{|c|}{$\mathrm{n}=47$} & \multicolumn{2}{|c|}{$\mathrm{n}=18$} & \multicolumn{2}{|c|}{$\mathrm{n}=65$} \\
\hline Compra & 17 & 36,2 & 7 & 38,9 & 24 & 36,9 \\
\hline Recolecta directa & 29 & 61,7 & 9 & 50,0 & 38 & 58,5 \\
\hline Otra & 3 & 6,4 & 2 & 11,1 & 5 & 7,7 \\
\hline Porque se consume C. pica & \multicolumn{2}{|c|}{$\mathrm{n}=47$} & \multicolumn{2}{|c|}{$\mathrm{n}=18$} & \multicolumn{2}{|c|}{$\mathrm{n}=65$} \\
\hline Nutritivo & 28 & 59,6 & 10 & 55,6 & 38 & 58,5 \\
\hline Buen sabor & 41 & 87,2 & 15 & 83,3 & 56 & 86,2 \\
\hline Afrodisíaco & 21 & 44,7 & 6 & 33,3 & 27 & 41,5 \\
\hline
\end{tabular}


TABLA 3

Aspectos socioeconómicos, captura y consumo de C. pica de acuerdo a la percepción de seguridad alimentaria

\begin{tabular}{|c|c|c|c|c|c|c|c|}
\hline \multirow[t]{2}{*}{ Características } & \multicolumn{2}{|c|}{ Seguro } & \multicolumn{2}{|c|}{ Inseguro } & \multicolumn{2}{|c|}{ Total } & \multirow[t]{2}{*}{$\mathbf{p}$} \\
\hline & $\mathbf{n}$ & $\%$ & $\mathbf{n}$ & $\%$ & $\mathbf{n}$ & $\%$ & \\
\hline Hacinamiento & \multicolumn{2}{|c|}{$\mathrm{n}=56$} & \multicolumn{2}{|c|}{$\mathrm{n}=67$} & \multicolumn{2}{|c|}{$\mathrm{n}=123$} & \\
\hline $\mathrm{Si}$ & 7 & 12,5 & 9 & 13,4 & 16 & 3 & $0,8783 * * *$ \\
\hline Ingresos mensuales & \multicolumn{2}{|c|}{$\mathrm{n}=56$} & \multicolumn{2}{|c|}{$\mathrm{n}=69$} & \multicolumn{2}{|c|}{$\mathrm{n}=125$} & \\
\hline Entre 0 y $\$ 200.000$ & 9 & 16,1 & 32 & 46,4 & 41 & 32,8 & $0,0003 * * *$ \\
\hline Entre $\$ 201.000$ y $\$ 408.000$ & 27 & 48,2 & 31 & 44,9 & 58 & 46,4 & $0,7140 * * *$ \\
\hline Entre $\$ 409.000$ y $\$ 600.000$ & 10 & 17,9 & 6 & 8,7 & 16 & 12,8 & $0,1274 * * *$ \\
\hline Entre $\$ 601.000$ y $\$ 800.000$ & 5 & 8,9 & 0 & 0,0 & 5 & 4 & \\
\hline Más de $\$ 800.000$ & 5 & 8,9 & 0 & 0,0 & 5 & 4 & \\
\hline Alimentación subsidiada & \multicolumn{2}{|c|}{$\mathrm{n}=57$} & \multicolumn{2}{|c|}{$\mathrm{n}=69$} & \multicolumn{2}{|c|}{$\mathrm{n}=126$} & \\
\hline $\mathrm{Si}$ & 15 & 26,3 & 17 & 24,6 & 32 & 25,4 & $0,8295 * * *$ \\
\hline Se consume C. pica en el hogar & \multicolumn{2}{|c|}{$\mathrm{n}=57$} & \multicolumn{2}{|c|}{$\mathrm{n}=69$} & \multicolumn{2}{|c|}{$\mathrm{n}=126$} & \\
\hline $\mathrm{Si}$ & 33 & 57,9 & 34 & 49,3 & 67 & 53,2 & $0,3345 * * *$ \\
\hline $\begin{array}{l}\text { La C. pica que consume se } \\
\text { adquiere por recolecta directa }\end{array}$ & \multicolumn{2}{|c|}{$\mathrm{n}=32$} & \multicolumn{2}{|c|}{$\mathrm{n}=33$} & \multicolumn{2}{|c|}{$\mathrm{n}=65$} & \\
\hline $\mathrm{Si}$ & 14 & 43,8 & 24 & 72,7 & 38 & 58,5 & $0,0178 * * *$ \\
\hline \multirow[t]{2}{*}{ Número de personas del hogar* } & \multicolumn{2}{|c|}{$\mathrm{n}=57$} & \multicolumn{2}{|c|}{$\mathrm{n}=69$} & \multicolumn{2}{|c|}{$\mathrm{n}=126$} & \\
\hline & \multicolumn{2}{|c|}{$4 \pm 2(3)$} & \multicolumn{2}{|c|}{$4 \pm 2(3)$} & \multicolumn{2}{|c|}{$4 \pm 2(3)$} & $0,8518 * *$ \\
\hline Años de escolaridad del jefe & & & & & & & \\
\hline del hogar* & & & & & & & \\
\hline & & $(6)$ & & $4(5)$ & 5,7 & $8(5)$ & $0,0014 * *$ \\
\hline Número de personas que & & & & & & & \\
\hline aportan ingresos $(\%)$ & & & & & & & \\
\hline 1 & 23 & 41,1 & 43 & 63,2 & 66 & 53,2 & $0,0138 * * *$ \\
\hline 2 & 25 & 44,6 & 18 & 26,5 & 43 & 34,7 & $0,0344 * * *$ \\
\hline 3 & 5 & 8,9 & 4 & 5,9 & 9 & 7,3 & $0,7620 * * *$ \\
\hline 4 & 3 & 5,4 & 3 & 4,4 & 6 & 4,8 & \\
\hline Cantidad de dinero destinado & & & & & & & $\mathrm{n}=126$ \\
\hline a alimentación* & 357 & 138540 & 267 & 143273 & 30917 & 47600 & \\
\hline & & 00) & & $00)$ & & & $0,0000^{* *}$ \\
\hline Producción de alimentos & & & & & & & \\
\hline $\mathrm{Si}$ & 15 & 26,3 & 18 & 26,5 & 33 & 26,4 & $0,9844 * * *$ \\
\hline Recolección de C. pica & & & & & & & \\
\hline $\mathrm{Si}$ & 15 & 26,3 & 24 & 34,8 & 39 & 30,9 & $0,3062 * * *$ \\
\hline
\end{tabular}


seguros $(\mathrm{p}=0,0178)$.

A partir del análisis de correspondencias múltiples se halló que algunas características de los hogares se perfilan alrededor de la seguridad alimentaria (figura 1 ), es así como en los cuadrantes inferiores se agrupan aquellos hogares seguros, con ingresos mayores a \$200.000 mensuales, no hacinados, compuestos por 2 ó 3 personas, con un hijo menor de 18 años, productores de alimentos para autoconsumo, con tipología nuclear completa o uniparentales madre, que vivían en casa propia o arrendada, con nivel educativo del jefe del hogar en secundaria o superior, ubicados en la localidad de Sapzurro, que manifestaron consumir C. pica pero no recolectarla. Por otro lado, en los cuadrantes superiores se agruparon aquellos hogares en inseguridad alimentaria, con ingresos menores o iguales a $\$ 200.000$ mensuales, que no producían alimentos para autoconsumo, compuestos por 4 a 10 integrantes, habitaban en vivienda prestada, en lo referente a la tipología eran familias mixtas, extensas o uniparentales padre, con nivel educativo del jefe del hogar primaria o ningún nivel, 2 ó más hijos menores de 18 años, ubicados en la localidad de Capurganá, que manifestaron no consumir C. pica pero si recolectarla.

\section{DISCUSIÓN}

El presente estudio mostró que los hogares de las localidades de Capurganá y Sapzurro tenían comprometida su seguridad alimentaria $(54,8 \%)$. Estos hogares se percibieron ligeramente más inseguros que el promedio de la región geográfica a la cual pertenecen, que es la Atlántica $(51,7 \%)$ y notablemente más inseguros que el promedio nacional $(40,8 \%)(5)$.

Esta situación de inequidad es mucho más profunda si se compara con las principales ciudades del país como son Bogotá, Cali y Medellín, donde la percepción de inseguridad alimentaria se ubica alrededor del 30\% (5). No obstante, al comparar la prevalencia de inseguridad alimentaria de Capurganá y Sapzurro con la observada en algunos países pobres como Bolivia (70.3\%) y Burkina Faso (73\%), esta se encontró más baja (17).

La situación de inseguridad alimentaria hallada en hogares de Capurganá y Sapzurro, concuerda con lo reportado en el estudio de Álvarez y cols. en el 2004 para el municipio de Acandí, donde se concluye que los hogares disponían de todos los grupos de alimentos, pero que ello no era condición suficiente para garantizar la seguridad alimentaria de sus integrantes dada la poca variedad de alimentos que conformaban cada grupo y la baja disponibilidad en gramos per cápita/día (3).

Se han identificado como factores de riesgo para la inseguridad alimentaria en hogares la disponibilidad, el acceso, utilización biológica y aceptabilidad de los alimentos, aspectos económicos, culturales, sociopolíticos, y sanitarios $(18,19)$. Para Capurganá y Sapzurro el principal factor asociado a la inseguridad alimentaria fue los bajos ingresos, el 91,3\% de los hogares inseguros manifestó ingresos iguales o menores a un salario míni-

\section{TABLA 4}

\section{Razones de disparidad para inseguridad alimentaria según características sociodemográficas y del recurso $C$. pica}

\section{Características}

\section{OR}

IC $95 \%$

Lim. Inf. $\quad$ Lim. Sup.

$\begin{array}{llll}\text { Ingresos mensuales } & 5,65 & 1,86 & 17,17 \\ \text { Número de años cursados } & 0,86 & 0,76 & 0,97 \\ \text { Localidad } & 1,44 & 0,53 & 3,92 \\ \text { Sexo del jefe del hogar } & 1,53 & 0,66 & 3,56 \\ \text { Recolección de C. pica } & 1,69 & 0,38 & 7,58 \\ \text { Venta de C. pica } & 0,33 & 0,05 & 1,94 \\ \text { Compra de C. pica } & 1,94 & 0,45 & 8,36 \\ \text { Consumo de C. pica } & 0,99 & 0,24 & 4,03\end{array}$

Ingresos: (1) inferior ó igual al mínimo; (0) superior al mínimo. Localidad: (1) Capurganá; (0) Sapzurro.

Sexo del jefe: (1) Mujer; (0) Hombre. Recolección: (1) Sí; (0) No. Venta: (1) Sí; (0) No.

Compra: (1) No; (0) Sí. Consumo: (1) Sí; (0) No. 


\section{FIGURA 1}

\section{Perfil de los hogares de Capurganá y Sapzurro, según características sociodemográficas, económicas, del recurso $C$. pica y de seguridad alimentaria}

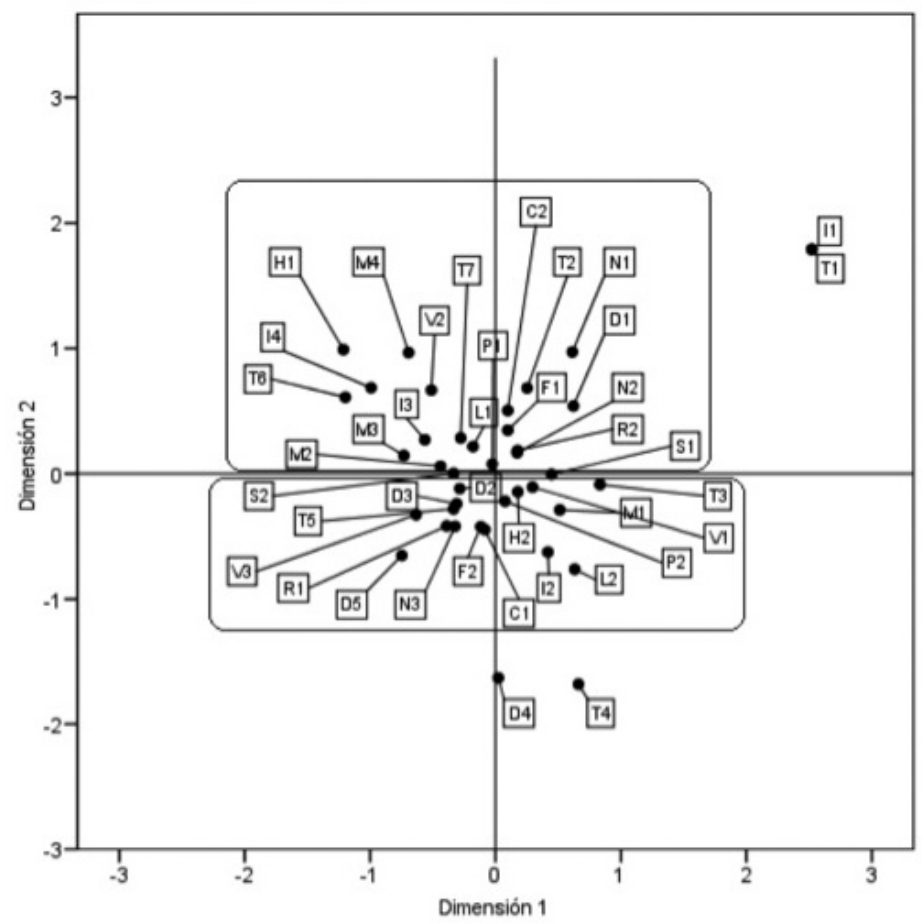

\section{Sigla Categoría de la variable}

L1 Localidad de Capurganá

L2 Localidad de Sapzurro

T1 Hogar unipersonal

T2 Hogar nuclear uniparental padre

T3 Hogar nuclear uniparental madre

T4 Hogar nuclear pareja sin hijos

T5 Hogar nuclear completa

T6 Hogar extenso

T7 Hogar mixto

S1 Jefatura del hogar femenina

S2 Jefatura del hogar masculina

V1 Vivienda propia

V2 Vivienda prestada

V3 Vivienda arrendada

D1 Ingresos entre $\$ 0$ y $\$ 200.000$

D2 Ingresos entre $\$ 201.000$ y $\$ 408.000$

D3 Ingresos entre $\$ 409.000$ y $\$ 600.000$

D4 Ingresos entre $\$ 601.000$ y $\$ 800.000$

D5 Ingresos mayores de $\$ 800.000$

P1 No produce alimentos

P2 Si produce alimentos

\section{Sigla Categoría de la variable}

C1 Si consume cigua

C2 No consume cigua

R1 No recolecta cigua

R2 Si recolecta cigua

F1 Hogar con inseguridad alimentaria

F2 Hogar con seguridad alimentaria

I1 1 integrante en el hogar

I2 2 a 3 integrantes en el hogar

I3 4 a 6 integrantes en el hogar

I4 7 a 10 integrantes en el hogar

H1 Hogar con hacinamiento

H2 Hogar sin hacinamiento

N1 Jefe del hogar sin ningún nivel educativo

N2 Jefe del hogar con nivel educativo primaria

N3 Jefe del hogar con nivel educativo secundaria/superior

M1 Hogar con 1 hijo menor de 18 años

M2 Hogar con 2 hijos menores de 18 años

M3 Hogar con 3 hijos menores de 18 años

M4 Hogar con 4 ó más hijos menores de 18 años 
mo (valor del 2006, $\$ 408000$ ), valor que al ser dividido por el número promedio de personas por hogar, daría una renta mensual bruta por persona aproximada a $\$ 81600$, lo que equivaldría a menos de dos dólares día.

Esta situación de pobreza puede ser entendida como una expresión de la marginalidad en que vive la mayor parte de la población de Capurganá y Sapzurro, un fenómeno estructural múltiple que describe cómo se integran o se excluyen los sectores sociales para el acceso a derechos fundamentales como la alimentación, la cual es percibida por los hogares en diferentes dimensiones, entre ellas la seguridad alimentaria (5), la cual tiene potenciales consecuencias en el estado nutricional, salud física, bienestar y calidad de vida de las personas $(17,18-21)$.

Es así como el aumento de los ingresos resultó ser un factor de protección para la inseguridad alimentaria en la zona de estudio, situación que concuerda con varios estudios en el ámbito mundial, en los cuales se encontró una relación inversa entre la inseguridad alimentaria y los ingresos, es así como una investigación realizada en Trinidad y Tobago, mostró que la inseguridad alimentaria era frecuente en hogares con bajos ingresos (77\%) (22), del mismo modo en Malasia se halló que la prevalencia de seguridad alimentaria fue de $77.4 \%$ en los hogares con los ingresos per cápita más altos (23).

El bajo nivel de escolaridad de la población fue el segundo factor asociado con la inseguridad alimentaria en el área de estudio. El trabajo de Álvarez y cols. en el 2004, mostró también un bajo nivel de escolaridad para el municipio de Acandí (3).

Los resultados del presente estudio revelan que el aumento en años de escolaridad del jefe del hogar es un factor de protección para la inseguridad alimentaria del hogar; similares resultados se hallaron en un estudio realizado en hogares pobres de una comunidad inglesa, en los cuales se encontró que la inseguridad alimentaria estaba asociada con bajos ingresos en el hogar y con bajo desarrollo educativo (24); no obstante, otras investigaciones no reportan asociación entre el bajo nivel educativo y la inseguridad alimentaria (23).

Tanto los ingresos como la educación han sido reportadas como variables claves en el control de los recursos del hogar, el poder en la toma de decisiones y consecuentemente en la salud y la supervivencia de los niños y del grupo familiar (25).

En Capurganá y Sapzurro, la cantidad de dinero destinada a alimentación fue otro factor relacionado con la inseguridad alimentaria, se encontró que la mayoría de los hogares gastaban poco en alimentación, pero esta cantidad representaba un porcentaje muy alto de sus ingresos totales, situación que fue evidenciada de forma similar por Oldewage-Theron y cols. en Sudáfrica (26).

El perfil de los hogares de Capurganá y Sapzurro, según características sociodemográficas, económicas y de seguridad alimentaria mostró que los hogares con bajos ingresos, compuestos por 4 a 10 integrantes, habitando en vivienda prestada, con bajo nivel educativo por parte del jefe del hogar, se perfilaban como hogares inseguros, situación que se asemeja a lo reportado en varios estudios. Es así como Rose y cols, hallaron que los hogares por debajo de la línea de pobreza o sin vivienda propia tenían más probabilidad de estar en insuficiencia alimentaria (27). Asimismo, se ha mostrado que los hogares compuestos por muchos integrantes están asociados con altas tasas de inseguridad alimentaria (20, 27-30), y el tener una secundaria completa por parte del jefe del hogar disminuye la probabilidad de insuficiencia alimentaria en el hogar (28).

En lo concerniente al recurso C. pica, la forma de adquisición del caracol se asoció con la seguridad alimentaria, donde los hogares inseguros adquirieron $\mathrm{C}$. pica esencialmente por recolección directa $(72,7 \%)$. De forma similar en la localidad de Cahuita en Costa Rica, Schmidt encontró que eran principalmente personas de bajos ingresos las que estaban involucradas en la recolección de C. pica (31).

Dado que C. pica posee un contenido de proteína del $23 \%$, este tiene el potencial para ser una buena fuente de dicho macronutriente para los hogares más vulnerables de las localidades del estudio. Sin embargo, dada la forma actual de uso del recurso en el área de estudio, este no impacta de manera importante la seguridad alimentaria de los hogares, probablemente por causas como el bajo consumo, el cual representa apenas un aporte del $1 \%$ a la recomendación de ingesta diaria de proteína; y los bajos ingresos que genera su venta, aproximadamente $\$ 40182$ anuales en promedio por hogar de recolector.

Para la zona de estudio, es claro que el bajo consumo C. pica no se debe a un problema exclusivo de acceso económico al recurso, también juega un papel importante su baja disponibilidad, la cual probablemente se relaciona con la notable disminución de la población de C. pica en los últimos años. Según la investigación de Osorno, el 100\% de los recolectores encuestados en Capurganá y Sapzurro manifestó que la abundancia de C. pica se ha reducido con relación al pasado (9).

La muerte masiva de C. pica causada periódicamente por descensos en la salinidad del agua (crecientes del río Atrato), la sobrepesca (9) y la muerte prematura de C. pica por captura antes de alcanzar la madurez sexual ( $<3 \mathrm{~cm}$ de diámetro de la concha) (7), son factores que han contribuido a la disminución de la población del 
recurso, con su consecuente efecto en la disponibilidad del mismo y en la seguridad alimentaria.

Actualmente no existe en el área de estudio ninguna regulación sobre el uso del recurso C. pica. La implementación de estrategias como tallas mínimas de captura $(>3 \mathrm{~cm})$ y periodos de veda en época de desove de $\mathrm{C}$. pica, tendrían un aporte considerable a la población del recurso $(7,9)$. La implementación de estas estrategias podría ayudar a la conservación del recurso, aumentar su disponibilidad y consumo, y mejorar su aporte a la seguridad alimentaria de los hogares vulnerables de la zona de estudio.

Por otra parte, es recomendable realizar estudios de factibilidad económica para el cultivo del caracol, el cual podría mejorar el impacto del recurso en la seguridad alimentaria de los hogares a la vez que se protege el recurso.

La forma actual de uso de C. pica no contribuye de manera importante a la Seguridad alimentaria de los hogares, aunque en un futuro podría ser una alternativa si se mejoran las condiciones de uso.

\section{RESUMEN}

Se describió la seguridad alimentaria en hogares de las localidades de Capurganá y Sapzurro y su relación con la utilización del recurso C. pica. Se realizó un estudio transversal a partir de una muestra de 126 hogares, representativa y con selección probabilística. Para la recolección de la información se utilizó la escala de percepción de seguridad alimentaria, las demás características se captaron por medio de una encuesta. En el análisis se utilizaron las pruebas chi cuadrado, $\mathrm{U}$ de Mann Whitney, razón de disparidades con sus intervalos de confianza y el análisis de correspondencias múltiple. El 54,8\% de los hogares se encontraron en inseguridad alimentaria. Esta se relacionó con bajos ingresos $(\mathrm{p}=0,0003)$, recolección directa del caracol $(\mathrm{p}=0,0178)$, escolaridad del jefe del hogar $(\mathrm{p}=0,0014)$ y cantidad de dinero destinado a alimentación $(\mathrm{p}=0,0000)$. El uso actual que se le da al caracol no contribuye de manera importante a la seguridad alimentaria de los hogares.

Palabras claves: Seguridad alimentaria, Cittarium pica, Capurganá, Sapzurro, Colombia.

Dirigir la correspondencia a:

Profesor

Gustavo Lozano Casablanca

Escuela de Nutrición y Dietética

Ciudadela Universitaria Robledo

Universidad de Antioquia.

Colombia.

Carrera $75 \mathrm{~N}^{\circ} 65-87$
Teléfono: (57-4) 2199200 - Fax: 2305007

E-mail: glozano@quimbaya.udea.edu.co

\section{BIBLIOGRAFÍA}

1. Food and Agriculture Organization. Rome Declaration World Food Security, World Food Summit. FAO. 1996.

2. Departamento Nacional de Planeación. Consejo Nacional de Política Económica Social-CONPES-. Política Nacional de Seguridad Alimentaria y Nutricional (PSAN), Documento Conpes 113. Bogotá; 2007.

3. Álvarez MC, Rosique J, Restrepo MT. Seguridad Alimentaria en los hogares de Acandí: La disponibilidad Alimentaria como indicador de la suficiencia alimentaria. Rev Chil Nutr 2004;31(3):318 - 29.

4. Ruiz M, Ruiz NY. La mortalidad por desnutrición infantil en Colombia. [Documento de Internet]. URL:http://www.unipiloto.edu.co/files/resourcesmodule/@random44edc86d04c8d/1180563214_ mortalidad.pdf. Fecha de consulta: 15 agosto 2008.

5. Instituto Colombiano de Bienestar Familiar. Encuesta nacional de la situación nutricional en Colombia, 2005. Catalina Borda Villegas ed. Bogotá: Panamericana Formas e Impresos; 2005.

6. Ministerio del Medio Ambiente de la República de Colombia. Resolución 0584. Bogotá; 2002. p 10.

7. Correa T. Algunos aspectos de la dinámica poblacional de la cigua Cittarium pica (Linnaeus, 1758) en el municipio de Acandí, Darién Caribe colombiano [Trabajo de grado para optar al título de Ecóloga de Zonas Costeras]. Turbo: Universidad de Antioquia; 2006.

8. Rosique J, Toro B, Correa T, Marín JG, Galeano N. Estudio ecológico y sociocultural de la Cittarium pica (Linnaeus, 1758) en el Darién Caribe colombiano. Medellín; 2007. p. 114.

9. Osorno AM. Bioecología de la Cigua o Burgao, Cittarium pica, en la costa continental del Caribe colombiano [Tesis de Grado]. Santa Marta: Universidad Jorge Tadeo Lozano; 2005.

10. Álvarez M, Estrada A, Montoya E, Melgar-Quiñonez M. Validación de la escala de seguridad alimentaria doméstica en Antioquia, Colombia. Salud Pública de México. 2006;48(6):474-81.

11. Lorenzana P, Mercado C. Measuring household food security in poor venezuelan households. Public Health Nutrition. 2002;5:851 - 7.

12. Wehler C, Scott R, Anderson J. The community childhood identification project: A model of domestic hunger-demonstration project in Seattle, Washington. J Nutr. 1992;24:29S-35S. 
13. Asociación Médica Mundial. La Declaración de Helsinki. [Página Web]. URL:http://www.wma. net/s/policy/b3.htm. Fecha de consulta: 16 octubre 2007.

14. Ministerio de Salud de la República de Colombia. Resolución 008430. Bogotá; 1992. pp 12.

15. Greenacre M. Correspondence analysis of the Spanish National Health Survey. Gac Sanit 2002;16 (2):160-70.

16. Aranha RN, Faerstein E, Azevedo GM, Werneck G, Lopes CS. Análise de correspondência para avaliação do perfil de mulheres na pós-menopausa e o uso da terapia de reposição hormonal Cuadernos Saúde Pública 200420 100-8

17. Melgar-Quinonez HR, Zubieta AC, MkNelly B, Nteziyaremye A, Gerardo MFD, Dunford C. Household Food Insecurity and Food Expenditure in Bolivia, Burkina Faso, and the Philippines. J Nutr 2006;136(5):1431S-7.

18. Campbell CC. Food Insecurity: A Nutritional Outcome or a Predictor Variable? J Nutr 1991; 121(3):408-15.

19. Instituto de Nutrición de Centro América y Panamá. [Página Web] URL:http://www.sica.int/incap/ san_breve.aspx? IdEnt=29. Fecha de consulta: 5 noviembre de 2008.

20. Rose D. Economic Determinants and Dietary Consequences of Food Insecurity in the United States. J Nutr 1999;129(2):517-520s.

21. Hamelin A-M, Habicht J-P, Beaudry M. Food Insecurity: Consequences for the Household and Broader Social Implications. J Nutr 1999;129(2):525-528s.

22. Gulliford M, Nunes C, Rocke B. The 18 Household Food Security Survey items provide valid food security classifications for adults and children in the Caribbean. BMC Public Health 2006;6(1):26.

23. Zalilah MS, Khor GL. Indicators and nutricional outcomes of household food insecurity among a simple of rural Malaysian women. Pakistan J Nutr 2004;3(1):50-5.

24. Tingay RS, Tan CJ, Tan NC-W, Tang S, Teoh PF, Wong R, et al. Food insecurity and low income in an English inner city. J Public Health 2003; 25(2):156-9.

25. Piwoz EG, Viteri FE. Syuding health and nutrition behavior by examining household decisión-making, intrahousehold resource distribution and the role of women in these processes. Food Nutr Bull 1985; $7: 1-31$.

26. Oldewage-Theron WH, Dicks EG, Napier CE. Poverty, household food insecurity and nutrition: Coping strategies in an informal settlement in the Vaal Triangle, South Africa. Public Health 2006 2006/9;120(9):795-804.

27. Rose D, Gundersen C, Oliveira V. Socio-Economic Determinants of Food Insecurity in the United States: Evidence from the SIPP and CSFII Datasets. Technical bulletin. Washington D. C.; 1998. pp. 1-20.

28. Alaimo K, Briefel RR, Frongillo EA, Jr, Olson CM. Food insufficiency exists in the United States: results from the third National Health and Nutrition Examination Survey (NHANES III). Am J Public Health 1998;88(3):419-26.

29. Olson CM, Rauschenbach BS, Frongillo EA, Kendall A. Factors Contributing to Household Food Insecurity in a rural upstate New York country. Fam Econ Nutr Rev 1997(10):2-17.

30. Mauldon J. Predicting Hunger and Overcrowding: How Much Difference Does Income Make? IRP Discussion paper. Madison - Wisconsin; 1996. pp. 1114-96.

31. Schmidt S, Wolff M, Vargas J. Population ecology and fischery of Cittarium pica (Gastropoda: Trochidae) en the Caribe coast of Costa Rica. Rev Biol Tropical 2002;50(3-4):1079-90. 